\title{
Evaluación pre-post del desarrollo psicomotor y el estilo de apego en usuarios de los Centros de Desarrollo Infantil Temprano
}

\section{Pre-post evaluation of psychomotor development and attachment style in users of Early Childhood Development Centers}

\author{
María Angélica Kotliarencoํㅗㄹ Esteban Gómez, Magdalena Muñoz \\ CEANIM, Chile \\ Iván Armijo \\ Universidad del Desarrollo, Chile
}

(Recepción: Octubre 2009 - Aceptación: Diciembre 2009)

\begin{abstract}
Resumen
Se presenta los resultados obtenidos por los Centros de Desarrollo Infantil Temprano (DIT) en familias con vulnerabilidad psicosocial. Mediante un estudio longitudinal no experimental, se observó una mejoría estadísticamente significativa en el desarrollo psicomotor infantil, identificándose en el EEDP un efecto preventivo para casos con normalidad inicial y un efecto nivelador para casos en riesgo inicial $(\mathrm{n}=166$, $\mathrm{p}<0.05$ ). Asimismo, se observó un aumento en la proporción de casos clasificados con apego seguro en el ADS y de disminución de aquellos con apego evitativo $(n=49$, $p<0.01)$. Si bien no pueden atribuirse vínculos causales con la intervención, los datos aportan evidencia que respalda la labor realizada por los Centros DIT e incentivan a desarrollar nuevas investigaciones en programas de intervención temprana en la realidad latinoamericana.

Palabras Clave: Intervención Temprana, Desarrollo Infantil, CEANIM, Evaluación de Resultados
\end{abstract}

\begin{abstract}
The outcomes achieved by the Centers of Early Child Development (DIT) in families with psychosocial vulnerability are presented. By means of a non experimental longitudinal study, a statistical significant improvement in infant psychomotor development was observed, identifying in the EEDP a preventive effect for the cases with initial normality and a leveling effect for the cases with initial risk ( $n=166, p<0.05)$. Likewise, it was observed an increase in the proportion of cases classified with a secure attachment in the ADS, and a decreased of those cases classified with evitative attachment $(n=49, p<0.01)$. Although causal links with the intervention cannot be established, data gives evidence that support the work of DIT Centers, and incentive to develop new research with early intervention programs in Latin-American reality.

Key Words: Early Intervention, Child Development, CEANIM, Outcomes Evaluation
\end{abstract}

1 Correspondencia: María Angélica Kotliarenco. CEANIM, Nueva de Bueras 180, Santiago de Chile. E-mail: mkotliarenco@ gmail.com 
En la actualidad, se encuentra firmemente establecida la importancia que tienen los primeros años de vida para todo el desarrollo humano, especialmente para los ámbitos del crecimiento cognitivo, emocional y social (Shonkoff \& Phillips, 2000; Schore, 2001; Lecannelier, 2006), si bien diversos autores han matizado los descubrimientos del programa de investigación en infancia temprana con la ponderación de las variables actuales, necesariamente sistémicas, que influyen en el curso del desarrollo para cada etapa del ciclo vital (Sroufe, Carlson, Levy \& Egeland, 1999).

También se han registrado notorios avances con respecto a la investigación sobre los factores de riesgo que dificultan que un niño o niña actualice y despliegue al máximo su potencial (Rutter, 1979; Appelyard, Egeland, van Dulmen \& Sroufe, 2005), y en el estudio y conceptualización de modelos teóricos que permitan comprender la interacción de estos factores en contextos ecológicos particulares, especialmente en el campo de la desprotección infantil (Cicchetti \& Toth, 1995; Glaser, 2000; Springer, Sheridan, Kuo \& Carnes, 2007). Entre los factores de riesgo más mencionados por la literatura se encuentran la pobreza, el maltrato infantil, la negligencia, el abandono, el abuso sexual, la deprivación sociocultural, el apego inseguro -particularmente de tipo desorganizado-, la depresión parental y el estrés familiar crónico (National Research Council, 1993). Actualmente, prima una concepción acumulativa y multi-sistémica de factores de riesgo por sobre una concepción unidimensional a la hora de explicar el peso específico de estos factores sobre el desarrollo infantil (Appelyard et al., 2005; Connell-Carrick, 2003).

A partir del estudio de los factores de riesgo y vulneración, se ha generado una corriente muy importante de investigación sobre los factores protectores y, más recientemente, de mecanismos de resiliencia que permiten comprender cómo es que niños, jóvenes, familias y comunidades afectadas por situaciones adversas logran superarlas e incluso salir transformadas y enriquecidas en el proceso (Rutter, 1993; Kotliarenco, Cáceres \& Fontecilla, 1997; Luthar, Cicchetti \& Becker, 2000; Cyrulnik, 2002). El enfoque de la resiliencia ha hecho aportes teóricos significativos para contrapesar la visión determinista y atomizadora del enfoque centrado en los riesgos y déficits, permitiendo estudiar desde múltiples disciplinas los procesos que permiten el cambio en el ser humano, en un continuo que incorpora desde el sustrato biológico más profundo (Kotliarenco \& Pardo, 2003; Cyrulnik, 2007), pasando por los procesos personales de duelo y resignificación (Cyrulnik, 2003), las dinámicas familiares de apoyo, pertenencia y protección (Walsh, 2004), hasta los espacios de narración y crecimiento que la comunidad y la cultura permiten al herido o al vulnerable para su reconstitución y proyección a nuevas posibilidades de futuro (Cyrulnik, 2008).

Recientemente, las líneas de investigación centradas en el desarrollo de la infancia temprana, y especialmente aquellas que han profundizado en los vínculos de contención y regulación que el ser humano va construyendo a lo largo del ciclo vital -bajo la teoría del apego-, han comenzado a converger con aquellas corrientes que han estudiado y teorizado sobre el fenómeno de las resiliencias (Kalawski \& Haz, 2003; Luthar, Cicchetti \& Becker, 2000), identificando como uno de los mecanismos de resiliencia por excelencia la constitución de un apego seguro (Kotliarenco \& Lecannelier, 2004). El cruce de estos modelos (de riesgo y vulnerabilidad, de protección y promoción del desarrollo humano, y de resiliencia personal, familiar y comunitaria) ha permitido pensar e implementar nuevas formas de apoyar y estimular a las familias en sus funciones de crianza, cuidado, protección y estimulación oportuna, siendo una de las formas más difundidas en países desarrollados los Programas de Intervención Temprana.

\section{Los Programas de Intervención Temprana y los Centros DIT}

Los Centros de Desarrollo Infantil Temprano (Centros DIT) fueron un proyecto piloto de intervención temprana, diseñado e implementado por el Centro de Estudios y Atención del Niño y la Mujer (CEANIM) en Chile durante los años 2006 a 2008, mediante financiamiento de la Fundación Integra. El proceso de diseño e implementación de los Centros DIT ha sido detallado en otras publicaciones (Leyton \& Ferrer, 2007; Palmeros, Rojas \& Traverso, 2008; Kotliarenco, Gómez \& Muñoz, 2009), por lo que aquí sólo se hará referencia a los elementos centrales del modelo. 
Los Centros DIT son programas de intervención temprana dirigidos a embarazadas a partir del quinto mes de gestación, y a familias con infantes hasta los 2 años de edad. Su objetivo apuntó a favorecer el desarrollo integral materno-infantil, por medio de intervenciones educativas, psicosociales y comunitarias coherentes con el marco teórico de la ecología humana, la resiliencia y el apego.

Si bien la coordinación del programa se sustentó desde un nivel central, se enfatizó a cada equipo la importancia de mantener un enfoque ecológicamente sensible a las realidades que surgieran en cada familia y comunidad. A partir de un análisis caso a caso, se ponderaban características de pobreza, vulnerabilidad, riesgo biopsicosocial o aislamiento de las redes locales, definiendo en conjunto con las participantes los servicios más pertinentes y adecuados a sus necesidades. Los servicios principales se encontraban definidos -talleres psicoeducativos y de autocuidado, visitas domiciliarias, espacios de estimulación y vinculación madre-hijo, cuidado ocasional del infante y derivación a las redes necesarias- operando con un modelo mixto que articuló servicios ofrecidos en el centro y servicios ofrecidos en el hogar de cada familia, siendo la visita domiciliaria el principal método de intervención utilizado (Kotliarenco, Muñoz, Gómez \& Aracena, 2009).

Una característica distintiva del proyecto fue la conformación de los equipos, ya que contempló el uso de para-profesionales o monitoras comunitarias (madres de la propia comunidad) debidamente capacitadas en diversas temáticas significativas para el adecuado ejercicio de sus funciones; cada centro DIT contó con 6 monitoras, supervisadas y apoyadas por un coordinador-profesional, contando además con personal administrativo que llevaba la gestión del centro.

\section{Tabla 1: Comparación entre las características del EHS, el Sure Start y los Centros DIT}

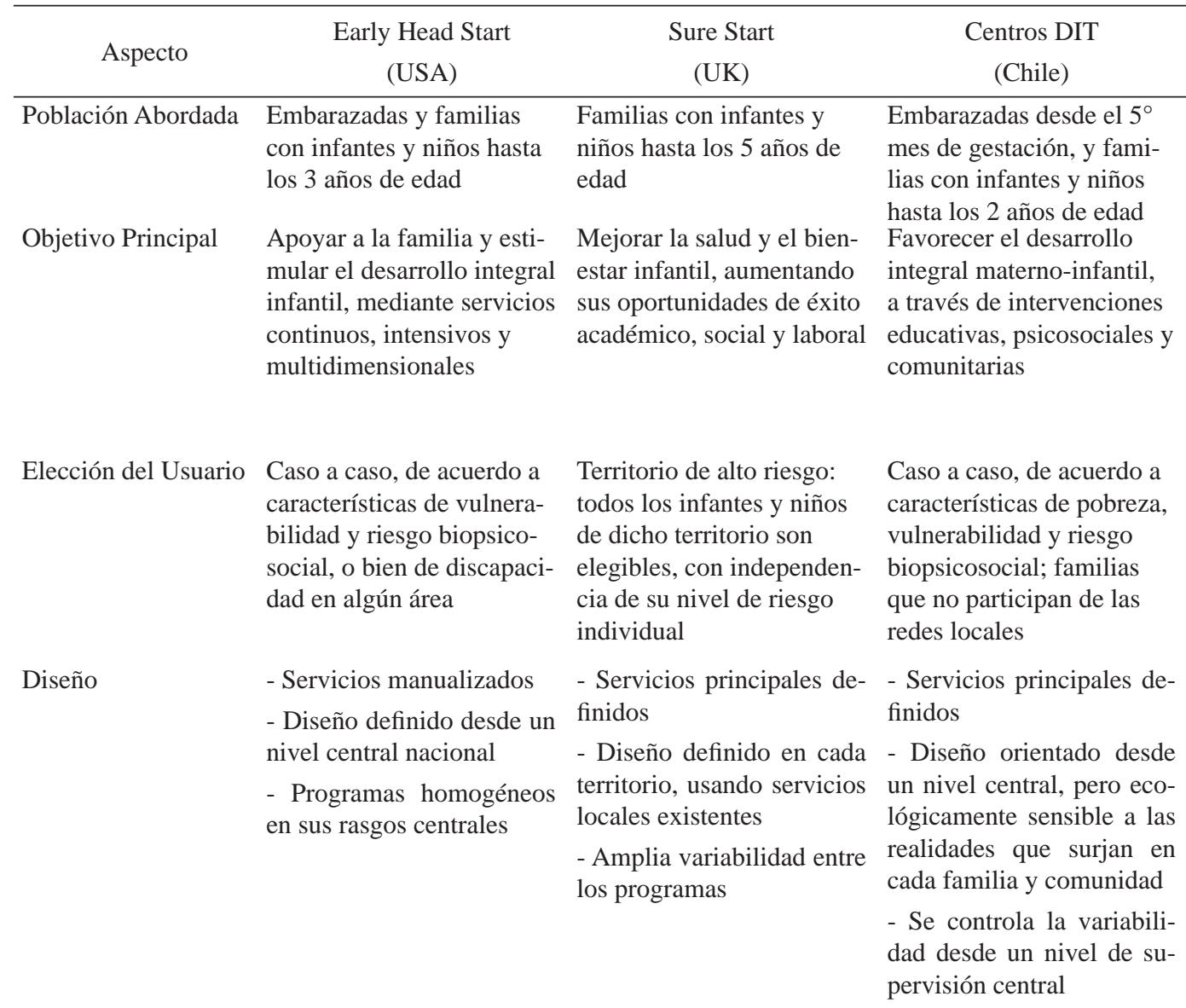




\begin{tabular}{llll} 
Modelo de servicios & $\begin{array}{l}\text { En el Centro, en el Hogar } \\
\text { o con un modelo mixto. }\end{array}$ & $\begin{array}{l}\text { Según las necesidades } \\
\text { detectadas por cada pro- } \\
\text { grama en el territorio }\end{array}$ & $\begin{array}{l}\text { Operan con un modelo } \\
\text { mixto Centro-Hogar }\end{array}$ \\
Recurso Humano & Profesionales & Profesionales & $\begin{array}{l}\text { Profesionales y Para- } \\
\text { profesionales (Monitoras } \\
\text { Comunitarias) }\end{array}$ \\
\hline
\end{tabular}

FUENTE: adaptado a partir de Kotliarenco, Gómez \& Muñoz (2009).

En la Tabla 1 se resumen las características de los modelos de intervención temprana más difundidos a nivel internacional y cómo se integraron esos componentes a los Centros DIT. Los Centros DIT se fundamentaron en los hallazgos de la literatura empírica sobre evaluaciones de diversos programas de intervención temprana, las que han puesto de relieve la importancia de contar con diseños claros, servicios de calidad, equipos competentes, debidamente capacitados y con un alto nivel de fidelidad al modelo, y usuarios involucrados y con participación activa, independientemente del foco de acción del programa (Love et al., 2006; Gray \& McCormick, 2005; Olds, Sadler \& Kitzman, 2007). Cuando estas condiciones están presentes, se ha encontrado que los niños intervenidos mejoran sus niveles cognitivos, emocionales y educacionales, y las relaciones e interacciones con sus figuras parentales entre otros hallazgos, con respecto a los grupos de control (Karoly et al., 1998, en Gray \& McCormick, 2005).

Por ejemplo, la evaluación nacional del programa Early Head Start en los Estados Unidos (Administration for Children and Families [ACF], 2002), benefició al grupo intervenido, obteniéndose diferencias estadísticamente significativas con respecto al grupo de control en las áreas de lenguaje, cognición y conducta, aunque la mayoría de los impactos correspondió a tamaños de efecto modestos (entre 10 y 20\%). Otros hallazgos relevantes fueron un mayor desarrollo socio-emocional en los niños y un incremento en las habilidades parentales, la autonomía parental y las interacciones padre-hijo (ACF, 2002). Por último, es importante iluminar el hallazgo de que los patrones de mayor impacto positivo se observaron más frecuentemente en programas EHS de diseño mixto (ACF, 2002), es decir, que incorporaban tanto servicios ofrecidos en el centro como servicios ofrecidos en el hogar.

Por otra parte, el programa Sure Start en Inglaterra ha tenido dos fases en su evaluación de resultados: la primera fase presentó resultados poco alentadores, principalmente asociados a la falta de rigurosidad del diseño del programa y por tanto, a la presencia de una gran variabilidad en las acciones realizadas por cada equipo de intervención (Rutter, 2006). Esta situación se revirtió en la segunda fase del Estudio de Impacto, demostrando una serie de beneficios en el grupo de intervención, a los tres años de edad, con respecto al grupo de control (NESS, 2008). Así, los niños viviendo en territorios con Programas Locales de Sure Start mostraron, en comparación al grupo de control, mejor desarrollo social, exhibiendo más conductas sociales positivas y una mayor auto-regulación e independencia. Asimismo, se observó una mejor competencia parental en las familias y el desarrollo de mejores ambientes hogareños de aprendizaje para sus hijos e hijas (NESS, 2008).

\section{Resultados preliminares de los Centros DIT}

En Chile, los Centros DIT fueron evaluados con respecto al proceso de implementación (Leyton \& Ferrer, 2007) y a los resultados obtenidos sobre la salud mental materna (Palmeros, Rojas \& Traverso, 2008). La evaluación de proceso realizada en 2007 abordó la percepción y valoración de las madres beneficiarias y de las propias monitoras comunitarias sobre su participación en los Centros DIT, el modelo de intervención, la relación operador-usuario, y los objetivos y resultados preliminares del proyecto, a través de grupos focales (Leyton \& Ferrer, 2007). Las conclusiones de esta evaluación de proceso señalan que "el diseño de esta iniciativa en cuanto a su propósito, objetivos y actividades resulta muy atingente a la realidad de su población objetivo, y puede entenderse desde la perspectiva de los actores involucrados, como una experiencia exitosa” (Leyton \& Ferrer, 2007, 
p. 52). Asimismo, se destaca que un aspecto central de la ayuda ofrecida por los equipos de los DIT ha sido el apoyo social y la psicoeducación de las madres y familias en temas relevantes para la crianza, desarrollo de competencias parentales y cuidados adecuados de los niños y niñas (Leyton \& Ferrer, 2007).

Un segundo estudio (Palmeros, Rojas \& Traverso, 2008) estuvo enfocado en evaluar el impacto de los Centros DIT sobre la depresión pre/post natal ( $n=75)$, en comparación con madres que asistían a controles obstétricos en centros de atención primaria, pero que no eran usuarias de los DIT (n=108), mediante la Escala de Depresión Post-Natal de Edimburgo. El grado de sintomatología depresiva inicial (pre-natal) no mostró diferencias significativas entre los DIT y los consultorios. Al evaluar el efecto de los DIT sobre las tasas de depresión post natal no se observaron diferencias significativas respecto al grupo de comparación; tampoco se registraron variaciones significativas en los niveles de depresión pre/post natal. Si bien la atención paliativa de la depresión materna no fue el objetivo de los Centros DIT, estos resultados mostraron que el modelo no sería necesariamente eficiente para abordar los problemas de salud mental de las madres, requiriendo una intervención especializada complementaria para abordar este aspecto. Una de las razones que esgrime el estudio mencionado para explicar estos resultados, es la baja derivación al sistema especializado en salud mental (42\% de las madres que lo requerían), si bien fue notoriamente superior a la tasa de derivación registrada en los consultorios (28\% de las madres que lo requerían) (Palmeros, Rojas \& Traverso, 2008).

A pesar de las valiosas contribuciones de los estudios señalados, los Centros de Desarrollo Infantil Temprano no han sido evaluados con respecto a sus objetivos principales, es decir, respecto a su efecto sobre el desarrollo infantil (motor, lingüístico y social) temprano, ni sobre la relación de apego cuidadora-infante. Este artículo busca contribuir a llenar esta carencia, presentando los hallazgos de una evaluación de resultados realizada en los Centros DIT, abordando los datos recopilados para el período de funcionamiento comprendido entre enero de 2006 y agosto de 2008.

\section{Método}

El presente estudio tuvo por objetivo describir características básicas de la población atendida en los DIT y evaluar los resultados obtenidos por la intervención con respecto al desarrollo psicomotor y el tipo de apego presente en los niños y en sus madres. Para ello, se utilizó un diseño no experimental longitudinal, con evaluaciones pre-post en la totalidad de los 15 centros implementados durante el período comprendido entre enero de 2006 y agosto de 2008.

\section{Instrumentos}

Cuestionario Socio-demográfico y de Riesgo Psicosocial: se diseñó una ficha de caracterización socio-demográfica y socioeconómica, que permitió registrar, además de datos básicos de identificación, una serie de factores de riesgo (empleo, pobreza, salud mental, estructura familiar, etc.) tanto en las madres ingresadas a los Centros DIT, como en el grupo familiar correspondiente.

Escala de Evaluación del Desarrollo Psicomotor de 0-24 meses (EEDP): esta escala evalúa desarrollo psicomotor en niños entre los 0 y 24 meses de edad. Es de aplicación individual y evalúa cuatro áreas del desarrollo: motora, lenguaje, social y coordinación, ello a través de la observación del niño y la aplicación de un cuestionario a la madre. La Escala consta de 75 ítems, y su aplicación dura aproximadamente 15 minutos. Entrega un perfil de desarrollo psicomotor para cada una de las áreas evaluadas, y un coeficiente de desarrollo. Este test ha sido estandarizado con niños chilenos y posee buenos índices de confiabilidad (test-retest, .70) y de validez de contenido (Rodríguez, Arancibia, Undurraga, 1976).

Escala Massie-Campbell o Escala de Apego Durante Stress (ADS): el ADS es un instrumento que se utiliza para evaluar la calidad de la relación vincular entre un cuidador y su bebé (0 a 18 meses), en situaciones de estrés del bebé (Massie \& Campbell, 1984). Fue creada para su uso rápido en el contexto de la consulta pediátrica, aunque también puede utilizarse en otros momentos de stress moderado del bebé (por ej., muda, alimentación o momentos breves de separación con un cuidador 
significativo). Es de fácil administración, corrección práctica y operativa; y fácil entrenamiento. Ha obtenido respaldo empírico sobre su confiabilidad y validez, mostrando una robusta confiabilidad inter-evaluador de .83 a .89 (Massie \& Campbell, 1984). Ha sido utilizada con poblaciones de NSE medio y bajo, y con poblaciones de alto riesgo. La pauta contiene 6 indicadores: mirada, vocalización, tacto, sostén, afecto y proximidad. Posee dos pautas de observación, una para la conducta del Cuidador y la otra para la conducta del Bebé. En cada uno de los indicadores se asigna un puntaje de 1 a 5, dependiendo del tipo de la frecuencia e intensidad del indicador.

\section{Procedimientos}

El proceso de recolección de datos fue parte integral del diseño de los Centros DIT desde un comienzo; así, se incorporó a las prácticas de los propios equipos la aplicación de todos los instrumentos de evaluación y el registro de los datos en formatos predefinidos por el equipo central de CEANIM; esta opción se tomó tanto por un tema de costos y recursos disponibles para la marcha del proyecto, como por opciones metodológicos.

El cuestionario de caracterización socio-demográfica se aplicó a todas las madres ingresadas al proyecto, totalizando 829 registros al primer semestre de 2006. La aplicación del test EEDP estuvo a cargo de los profesionales de cada centro DIT, mientras que la aplicación del ADS estuvo a cargo de las propias monitoras comunitarias, quienes recibieron la debida capacitación inicial, lo que se reflejó en los altos índices de confiabilidad obtenidos. Tanto el test EEDP como el test ADS fueron aplicados al inicio del proceso de intervención y al cierre del mismo, con un intervalo aproximado de 18 meses.

Los resultados de ambas pruebas y del cuestionario de caracterización fueron registrados en fichas de formato único, las cuales fueron recopiladas por el equipo central de coordinación de CEANIM al término de cada período de aplicación. Una vez recopiladas fueron digitadas en una base de datos única, siendo la fuente de información usada en el presente estudio; desde el punto de vista de los resguardos éticos, este estudio tomó todas las medidas necesarias para cautelar la confidencialidad y el tratamiento riguroso de los datos consignados en los registros de la institución.

\section{Análisis de Datos}

Para el análisis del test EEDP fue necesario realizar una depuración de datos, filtrando de la muestra aquellos casos que no contasen con una medición inicial y una final. Esto implicó una importante pérdida muestral, ya que de los 620 infantes en los cuales se registró alguna medición, se contó finalmente con un total de 166 casos (26,8\%) que registran una medición pre y también una medición post.

La aplicación del test ADS para medir el apego, involucró una serie de chequeos inter-observadores con el fin de poder determinar la validez de la aplicación. Así, de las 478 aplicaciones en el primer punto de medición, 329 (68,8\%) pueden ser consideradas válidas, mientras que las 149 restantes (31,2\%) se consideran dudosas. De las 162 aplicaciones post-intervención, 148 (91,4\%) son consideradas válidas, y solo 14 (8,6\%) son dudosas. Si bien se redujo la cantidad de aplicaciones en la segunda medición, la cantidad de aplicaciones válidas aumentó notoriamente. Para este estudio se observó, en aquellos casos que cumplieron con los estándares óptimos de aplicación del instrumento, una confiabilidad medida con el alfa de Cronbach de .737 al inicio y .736 al final, para la pauta de los infantes; y .839 al inicio y .824 al final, para la pauta de las madres, lo que confirma los adecuados niveles de confiabilidad del instrumento. El análisis de la confiabilidad de aplicación por centro muestra que la mayoría de las aplicaciones registradas son confiables y que se distribuyen en forma relativamente homogénea entre los 15 centros DIT.

Como los sujetos evaluados en la primera y la segunda medición con el test ADS no fueron necesariamente los mismos, se realizó una sub-muestra con los casos que tenían ambas mediciones, identificándose 97 casos, de los cuales 49 casos pasaron todas las revisiones y pueden considerarse confiables en ambos tiempos. Esta sub-muestra se utilizó para contrastar los hallazgos encontrados en la muestra general. 
Los datos se sometieron a análisis descriptivos y de frecuencia para las características sociodemográficas y los estados iniciales en el desarrollo psicomotor y apego de los niños, al momento de ingresar a los Centros DIT. Posteriormente, se realizaron análisis comparativos entre los puntos de medición del inicio y término del proceso de intervención de los Centros DIT.

\section{Resultados}

\section{Características familiares}

Los DIT cubrían al momento del ingreso de las díadas un total de 4.226 personas, con un promedio de 5,5 integrantes y en un rango de entre 2 y 18 personas por hogar $(\mathrm{n}=768)$. Del total de personas que percibían ingresos en los grupos familiares (n=1876), solamente un 19,5\% tenía contrato indefinido (ver Tabla 2), lo que muestra una elevada inestabilidad laboral. Concordantemente, la mayoría de las familias mostraba precariedad económica, lo que se refleja en el ingreso familiar líquido mensual, que promedió \$188.980; así, el 40\% de las familias vivía con menos del sueldo mínimo al año 2006. Respecto a la previsión de salud de las madres ingresadas a los DIT (n=639), la inmensa mayoría se encontraba inscrita en FONASA (98,3\%), registrándose un mayoritario 68,2\% que lo hacía en el fondo A, un 14,7\% en el B, un 9,1\% en el fondo C y un 6,3\% en el fondo D²; nuevamente, estos datos refuerzan la imagen de que la familia promedio atendida en los Centros DIT vivía en situación de pobreza o incluso de indigencia al momento del ingreso.

La edad de las madres fluctuó entre 13 a 44 años de edad, con una media de 24,3 años y una distribución normal, mientras que los padres biológicos de los niños ingresados $(n=729)$, registraron una edad promedio de 26,7 años de edad, con un rango entre 14 y 62 años. Respecto a su escolaridad ( $n=779$ ), se destaca que la gran mayoría no completó sus estudios, con solo un 31,8\% que tenía educación media completa o superior. Al analizar el estado civil $(n=713)$, se observó que el matrimonio se daba en una minoría de los casos (22,3\%).

Respecto a la etapa en que ingresaron al programa, 51,1\% correspondió al período prenatal y $48,9 \%$ al posnatal, siendo en un 52,5\% madres primigestas. Del grupo con ingreso prenatal, el mes de gestación promedió 6,1 meses, cumpliéndose el criterio de focalización del programa.

Tabla 2: Características de la Población atendida en los Centros DIT al año 2006

\begin{tabular}{lcccc}
\hline Indicador al ingreso & Promedio & Desv. Estándar & $\mathrm{N}$ & $\%$ \\
\hline Integrantes por Hogar & 5,5 & 2.51 & & \\
Ingreso Mensual Fliar. & $\$ 188.980$ & 135.880 & & \\
Estabilidad Laboral & & & \\
$\quad$ Contrato a plazo & & 623 & 33,2 \\
$\quad$ Contrato indefinido & & 365 & 19,5 \\
$\quad$ Sin Contrato & & & 888 & 47,3 \\
\hline Mes Gestación (grupo prenatal) & 6,1 meses & 1.61 & 405 & 48,9 \\
Edad Infantes (grupo posnatal) & 2,59 meses & 3.75 & 423 & 51,0
\end{tabular}

2 El grupo A es para aquellas personas carentes de recursos o indigentes; el grupo B es para aquellas cuyo ingreso imponible mensual sea menor o igual a $\$ 165.000$; el grupo C es para quienes su ingreso imponible mensual sea mayor a $\$ 165.000$ y menor o igual a \$240.900; mientras que el grupo D es para aquellas personas cuyo ingreso imponible mensual sea mayor a $\$ 240.900$ (extraído de www.fonasa.cl). 


\begin{tabular}{|c|c|c|c|c|}
\hline Edad Padres & 26,7 años & 7.42 & & \\
\hline Edad Madres & 24,3 años & 6.83 & & \\
\hline 18 años o menos & & & 174 & 21,0 \\
\hline 19 - 30 años & & & 490 & 59,1 \\
\hline $31-40$ años & & & 145 & 17,5 \\
\hline 41 años o más & & & 20 & 2,4 \\
\hline Escolaridad Madres & 9,54 años & 3.22 & & \\
\hline Básica & & & 267 & 34,3 \\
\hline Media incompleta & & & 184 & 23,6 \\
\hline Media completa & & & 202 & 25,9 \\
\hline Superior & & & 46 & 5,9 \\
\hline Otros & & & 80 & 10,3 \\
\hline \multicolumn{5}{|l|}{ Estado Civil Madres } \\
\hline Casadas & & & 159 & 22,3 \\
\hline Convivencia & & & 309 & 43,3 \\
\hline Pareja no conviviente & & & 59 & 8,3 \\
\hline Separada & & & 23 & 3,2 \\
\hline Soltera & & & 119 & 16,7 \\
\hline
\end{tabular}

\section{Características de los niños}

Respecto a los niños y niñas atendidos, se registraron datos para un total de 535 nacidos al momento del ingreso de sus madres al programa. Su edad al ingreso fue de 2,59 meses en promedio, con un mínimo de 0 y un máximo de 20 meses. El sexo de los infantes se distribuyó en 53,1\% masculino y $46 \%$ femenino.

Al ingreso, se observó que los niños presentaron un desarrollo psicomotor dentro de parámetros normales para el $86,8 \%$ de los casos, mientras que $12,4 \%$ se ubicó en situación de riesgo y solo 0,8\% presentó indicadores de retraso en el EEDP. Asimismo, se observó que los estilos de apego predominantes en casi todas las categorías fueron el apego seguro (8,2 - 52,7\% de incidencia) y, en segundo lugar, el apego evitativo (3,0 - 38,4\% de incidencia). Los resultados en general reflejan lo esperable en las codificaciones generales de apego, con cerca de la mitad de los casos mostrando un apego de tipo seguro.

\section{Cambios observados en el Desarrollo Psicomotor de los Niños}

A nivel general, se registraron cambios esperables en las variables de edad mental $(t(165)=50,94$, $\mathrm{p}<0.05)$ y edad cronológica $(t(165)=44, \mathrm{p}<0.05)$. No se registraron diferencias en la relación entre edad cronológica y mental $(t(165)=-1,57, \mathrm{p}<0.05)$, ni en los puntajes estándar $(t(165)=-0,432$, $\mathrm{p}<0.05)$, lo que da cuenta de un crecimiento armónico en los niños participantes de la intervención. 
Tabla 3: Tamaño de efecto y potencia de prueba de las diferencias encontradas en el EEDP

\begin{tabular}{|c|c|c|c|c|}
\hline \multirow{3}{*}{$\begin{array}{l}\text { Escalas del } \\
\text { EEDP }\end{array}$} & Medición Pre & Medición Post & \multirow{3}{*}{ Tamaño de Efecto } & \multirow{3}{*}{ Potencia de Prueba } \\
\hline & Promedio & Promedio & & \\
\hline & Des. Típ. & Des. Típ. & & \\
\hline \multirow{2}{*}{ Coordinación } & 34,52 & 66,51 & \multirow{2}{*}{0.29} & \multirow{2}{*}{0.98} \\
\hline & 16.51 & 6.38 & & \\
\hline \multirow{2}{*}{ Social } & 32,88 & 65,68 & \multirow{2}{*}{0.27} & \multirow{2}{*}{0.96} \\
\hline & 16.46 & 7.86 & & \\
\hline \multirow{2}{*}{ Lenguaje } & 34,21 & 63,96 & \multirow{2}{*}{0.30} & \multirow{2}{*}{0.99} \\
\hline & 16.28 & 7.12 & & \\
\hline \multirow{2}{*}{ Motor } & 32,83 & 66,39 & \multirow{2}{*}{0.28} & \multirow{2}{*}{0.98} \\
\hline & 15.14 & 6.65 & & \\
\hline
\end{tabular}

Al finalizar la intervención, se detectaron aumentos significativos en los puntajes de todas las escalas EEDP, con un promedio de mejoría de 30 puntos aproximadamente; así, la mejoría fue de 29.8 puntos promedio para la escala de lenguaje $(t(162)=28,84, \mathrm{p}<0.05), 31.9$ puntos promedio para la escala de coordinación $(t(162)=31,98, \mathrm{p}<0.05), 32.7$ puntos promedio para la escala de habilidades sociales $(t(162)=30,78, \mathrm{p}<0.05)$, y 33.6 puntos promedio para la escala de habilidades motoras $(t(162)=35,45, \mathrm{p}<0.05)$. En general, la mejoría esperada fluctúa entre 30 y 33 puntos, con un nivel de confianza de 0.95 . Los puntajes de ambas mediciones se muestran altamente correlacionados, lo que permite suponer que la distribución interna de los datos se mantiene relativamente estable para los niños evaluados.

$\mathrm{Al}$ analizar el comportamiento de las escalas en cada medición, se puede observar un patrón constante en las escalas, de aumento de los puntajes y reducción de la heterogeneidad en la segunda medición. Esto indica que los grupos se homogenizan, mostrando patrones de desarrollo más comunes entre los niños después de la intervención. Las diferencias en las desviaciones estándar resultan en tamaños de efecto reducidos en la comparación de los puntajes, pero sin dañar la potencia de la prueba, lo que se traduce en que el cambio observado es confiable y en la línea de un aumento de puntaje con homogenización inter-sujeto post-intervención de los Centros DIT.

Gráfico 1: Nivel de desarrollo psicomotor Pre-Post intervención de niños del centro DIT,según el test EEDP

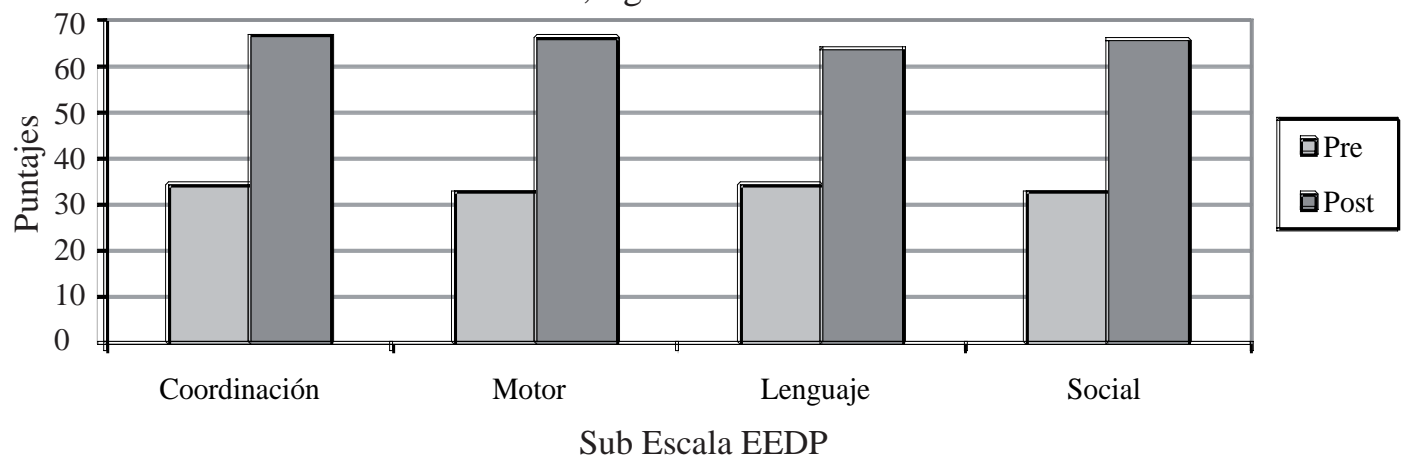

Asimismo, se observó un efecto positivo de la intervención en los casos de riesgo detectados, entre la clasificación general de desarrollo psicomotor pre en el test EEDP y la clasificación general post. En este aspecto, del total de casos clasificados en situación de Riesgo con anterioridad a la 
intervención de los Centros DIT, un 85.7\% ( $\mathrm{n}=18)$ mejoró hasta alcanzar criterios de normalidad en su desarrollo psicomotor, quedando solamente un 9.5\% clasificados en condición de retraso $(n=2)$ y un $4.8 \%$ que se mantuvo en condición de riesgo $(n=1)$. Se puede hablar entonces de un impacto positivo de la intervención en la recuperación de casos de riesgo detectados.

Por su parte, del total de casos que fueron clasificados en el grupo de Desarrollo Normal al inicio del proceso $(\mathrm{n}=148)$, un $6.6 \%$ fueron clasificados en condición de riesgo al finalizar la intervención ( $\mathrm{n}=10)$ y solamente un $1.4 \%$ en condición de retraso en el desarrollo $(\mathrm{n}=2)$, manteniéndose el 91.9\% de los niños y niñas en condición de normalidad ( $n=136)$. En este sentido, los casos que empeoraron pueden ser considerados parte de la variación estadística esperada por el paso del tiempo, dado que los problemas de desarrollo son más fácilmente detectables a medida que los niños y niñas crecen. El detalle de la variación puede observarse en la tabla 4.

Tabla 4: Cambio observado en la clasificación de desarrollo psicomotor según EEDP antes y después

\begin{tabular}{|c|c|c|c|c|c|c|c|c|c|}
\hline & & \multicolumn{8}{|c|}{$\begin{array}{c}\text { Desarrollo Psicomotor } \\
\text { Evaluación Post }\end{array}$} \\
\hline & & Normal & $\%$ & Riesgo & $\%$ & Retraso & $\%$ & Total & $\%$ \\
\hline Desarrollo Psicomotor & Normal & 136 & 91.9 & 10 & 6.6 & 2 & 1.4 & 148 & 100 \\
\hline \multirow[t]{2}{*}{ Evaluación Pre } & Riesgo & 18 & 85.7 & 1 & 4.8 & 2 & 9.5 & 21 & 100 \\
\hline & Total & 154 & & 11 & & 4 & & 169 & \\
\hline
\end{tabular}

\section{Cambios observados en las Conductas de Apego de los Niños}

Al observar las codificaciones hechas posterior a la intervención de los Centros DIT, se identificó un cambio en la distribución de los tipos de apego, con un aumento del estilo de apego seguro en todas las escalas, con entre un 37,8\% y un 72,3\%, y una notable disminución del estilo de apego evitativo, con entre un $2 \%$ a un $31,1 \%$. Es interesante notar que en la aplicación post-intervención, se observó un aumento del estilo ansioso en las conductas observadas en los niños, en las distintas categorías (entre 2,7\% y 25,9\% según la categoría), manteniéndose la dimensión Tacto B con un predominio del estilo de apego Ansioso (45,9\%), lo cual podría estar dando cuenta de un patrón propio de la actividad evaluada.

Gráfico 2: Resultados Pre-Post del Estilo de Apego en los Niños, según las escalas del test $A D S$

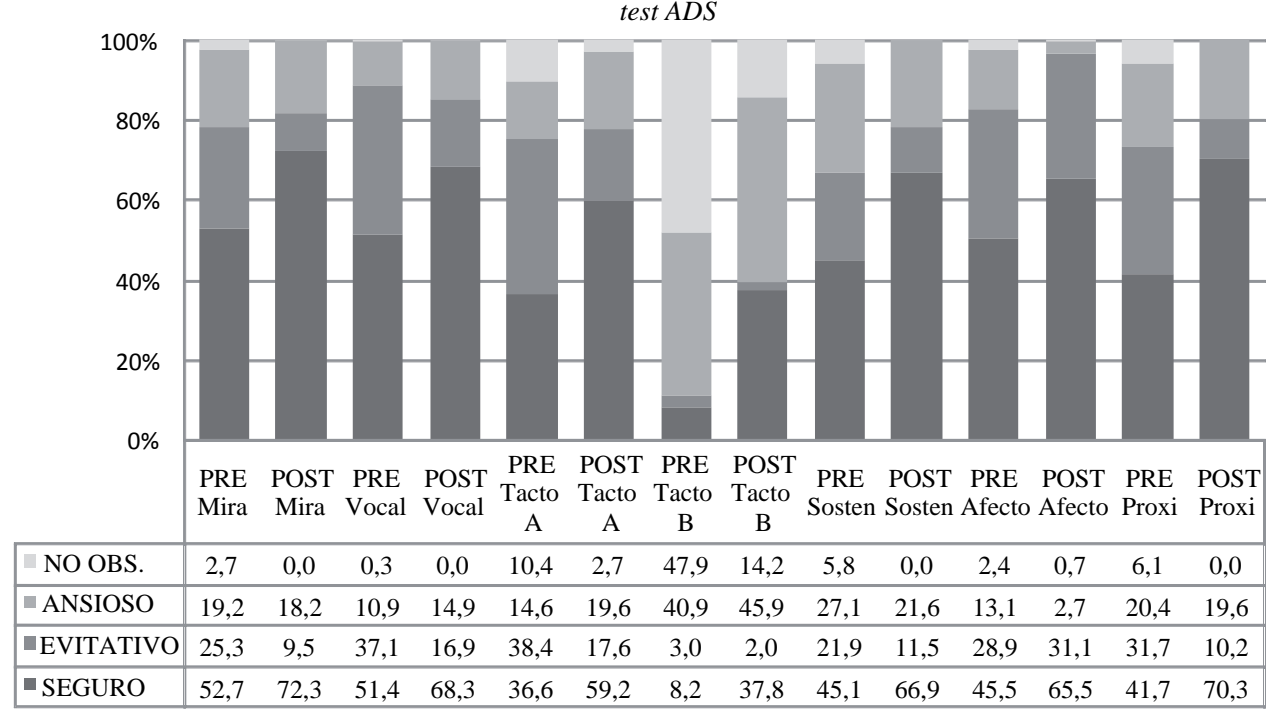


Se detectó un claro patrón en las diferencias encontradas, con un aumento estadísticamente significativo del estilo de apego seguro $(\mathrm{p}<0.01)$ y una disminución estadísticamente significativa del estilo de apego evitativo y de la clasificación No Observado $(\mathrm{p}<0.01)$. El estilo de apego ansioso, si bien registra cambios, no son estadísticamente significativos, salvo en la disminución observada en la categoría Afecto, el que se vuelve menos ansioso en la medición post (ver gráfico 2). Los análisis realizados con la sub-muestra de 49 casos que registraron aplicaciones pre-post confiables para ambas mediciones, confirman la tendencia previamente detectada, pasando de estilos de apego ansioso $\mathrm{y}$ apego evitativo a estilos de apego seguro en todas las categorías $(\mathrm{p}<0.01)$, salvo las que tienen que ver con la dimensión Tacto B.

\section{Cambios observados en las Conductas de Apego de las Madres}

$\mathrm{Al}$ observar las conductas de las madres se ve un comportamiento similar al observado en los niños y niñas; así, se registra una tendencia al aumento del estilo de apego seguro en todas las categorías de análisis entre la medición pre y la medición post-intervención. Al analizar en busca de diferencias estadísticamente significativas, se observó que se repite el patrón detectado en los niños de un aumento del estilo de apego seguro de manera estadísticamente significativa en todas las categorías ( $<<0.01)$, salvo en la expresión de Afecto. Cabe destacar, sin embargo, que en esa categoría existía un nivel muy alto de apego seguro desde antes de la intervención, de modo que si bien se registra un aumento, este no alcanza a ser significativo, probablemente producto del alto nivel inicial (ver gráfico 3).

El análisis de cambio de los casos en los que se cuenta con mediciones confiables en ambas ocasiones ( $\mathrm{n}=49$ ) ratifican lo anteriormente dicho, observándose una transición hacia un estilo de apego seguro en la mayoría de las categorías ( $\mathrm{p}<0.01$ ). Nuevamente, si bien no se puede sacar conclusiones definitivas, debido a la falta de un grupo de control (lo que mantiene como hipótesis alternativa que el cambio pueda ser consecuencia de procesos de maduración propios de la maternidad), sí es claro que la tendencia es muy marcada y que, en términos generales, termina con una distribución de apego seguro muy superior a lo esperable estadísticamente en la población normal

Gráfico 3: Resultados Pre-Post del Estilo de Apego en las Madres, según las escalas del test ADS

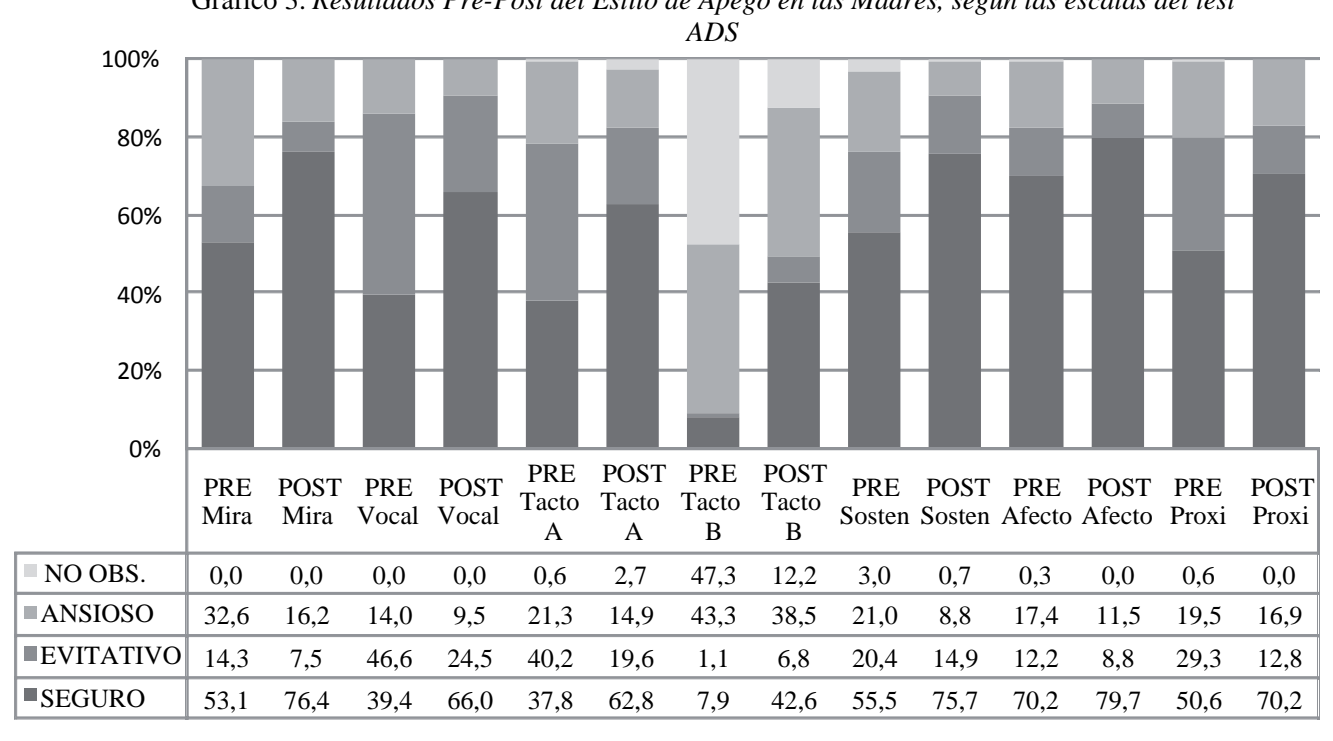




\section{Discusión y Conclusiones}

A pesar del terreno conquistado por la investigación interdisciplinaria y el ambicioso programa de las neurociencias, no ha sido sencillo desarrollar programas de intervención temprana indiscutiblemente exitosos (Shonkoff \& Phillips, 2000; Kotliarenco, Gómez \& Muñoz, 2009). La implementación de estas iniciativas se ha visto obscurecida por diversos problemas metodológicos y de diseño, lo que ha implicado que muchas decisiones de política pública sean tomadas sobre la base de experiencias anecdóticas, relegando a un segundo plano el uso de la práctica basada en evidencia (Olds, Sadler \& Kitzman, 2007). Estos problemas no son ajenos al contexto latinoamericano, donde se ha criticado fuertemente la notoria carencia de evaluación de los programas dirigidos a la infancia temprana que se adecúen a los desafíos y características particulares de cada país (Strasser, 2006; Bedregal, 2006; Lecannelier, Hoffman, Flores \& Ascanio, 2008).

Los Centros de Desarrollo Infantil Temprano pueden considerarse un aporte en esa dirección, por cuanto se trata de un modelo de atención desarrollado en Chile por CEANIM sobre la base tanto de los aprendizajes internacionales como de la propia experiencia acumulada (Kotliarenco, Gómez \& Muñoz, 2009). En línea con la teoría ecológica del desarrollo humano, la teoría del apego, y el enfoque de la resiliencia personal, familiar y comunitaria (Shonkoff \& Phillips, 2000; Schore, 2001; Rutter, 1993; Kotliarenco, Cáceres \& Fontecilla, 1997; Luthar, Cicchetti \& Becker, 2000; Cyrulnik, 2002), los DIT fueron concebidos como una forma de apoyar a las familias más vulnerables y aisladas de la red formal de apoyo, en la crianza y adecuada estimulación de sus hijos e hijas, desde la gestación hasta los dos primeros años de edad.

El presente estudio arroja valiosa información que permite confirmar que el programa se focalizó en la población para la cual fue diseñado. Así, la caracterización mostró que los Centros DIT atendieron a familias pobres, con inestabilidad laboral, bajos ingresos económicos y escasa protección social. La usuaria promedio fue una madre joven, con baja escolaridad, alto nivel de depresión pre y posnatal (Palmeros, Rojas \& Traverso, 2008) y un estado civil mayoritariamente caracterizado por la convivencia no formalizada. La población de mujeres atendidas se distribuyó equitativamente entre madres esperando su primer hijo y madres que ya tenían hijos anteriores; asimismo, la mitad ingresó en el último trimestre de la etapa pre natal y la mitad en el primer trimestre de la etapa pos natal, lo que da cuenta de la validez de los criterios de focalización del programa.

Los principales indicadores de resultado del modelo DIT apuntaron al fortalecimiento del desarrollo infantil temprano en el ámbito de las habilidades psicomotoras, sociales y lingüísticas, y a la estimulación de un estilo de apego seguro entre las madres y sus hijos (Kotliarenco, Gómez \& Muñoz, 2009). En consideración a estos aspectos, el presente estudio aporta evidencia en el logro de los objetivos descritos; respecto al desarrollo psicomotor, se observó una mejoría estadísticamente significativa en el EEDP de aproximadamente 30 puntos en todas las escalas, identificándose un efecto preventivo para los casos con normalidad inicial y un efecto nivelador para los casos en situación de riesgo inicial. Esto quiere decir que aun tratándose de población altamente vulnerable (como queda reflejado en la caracterización de las familias atendidas), donde sería esperable un notorio efecto negativo del entorno sobre los parámetros de desarrollo infantil (Rutter, 1979; Appelyard, Egeland, van Dulmen \& Sroufe, 2005), el modelo DIT lograría mantener la ventaja inicial observada en los bebés que califican en su desarrollo psicomotor dentro del rango normal. En aquellos pocos casos que en sus primeros meses de vida se detectó un rezago en el desarrollo, el modelo DIT tendría una influencia positiva favoreciendo su nivelación hacia parámetros de normalidad.

Asimismo, se observó una marcada tendencia, tanto en los niños como en las madres, de aumento en la proporción de casos clasificados con apego seguro en el ADS y de disminución de aquellos clasificados con apego evitativo, tras la intervención de los Centros DIT. Si bien no puede atribuirse causalidad debido a la falta de un grupo de control (siendo una limitación metodológica de este estudio), los datos aportan evidencia que respaldaría la labor realizada por los Centros DIT en las variables estudiadas, es decir, sobre el desarrollo psicomotor y el apego infantil. 
Estos resultados se potencian con los obtenidos por la evaluación cualitativa realizada por Leyton \& Ferrer (2007), la cual concluyó que la experiencia era percibida como exitosa por parte de los propios actores involucrados (monitoras comunitarias y madres usuarias) con respecto al cumplimiento de sus objetivos declarados. Al profundizar en las variables que pudiesen explicar los buenos resultados de los Centros DIT, es posible identificar como componentes centrales el apoyo social cotidiano, la psicoeducación de las madres y familias en tópicos significativos para el desarrollo infantil y el desarrollo de las competencias parentales en las madres (Leyton \& Ferrer, 2007). Asimismo, una variable identificada en este estudio que podría ser relevante para el logro parcialmente demostrado, fue el alto porcentaje de adherencia registrado para todo el proceso de intervención, siendo cercano al $75 \%$ de los casos.

Por otra parte, si bien un estudio previo sobre la efectividad del modelo DIT no detectó efectos significativos sobre la salud mental materna (Palmeros, Rojas \& Traverso, 2008), un estudio reciente que evaluó los programas de intervención temprana Early Head Start, mostró que los efectos sobre la salud mental materna -específicamente la depresión en las madres- no eran detectables inmediatamente, sino que una vez transcurridos cerca de dos años con posterioridad a la intervención (Chazan-Cohen et al., 2007). En este sentido, resulta imperativo incentivar el desarrollo de nuevas investigaciones en programas de intervención temprana en la realidad latinoamericana, por ejemplo, que permitan identificar efectos a mediano y largo plazo, y no solo al cierre de los procesos de intervención.

Un aspecto que resulta fundamental estudiar en profundidad, es el uso de profesionales y paraprofesionales entrenados en modelos específicos de intervención temprana, ya que se requiere con urgencia levantar evidencia que respalde y de sustento a formas de trabajo adecuadas al contexto de los países latinoamericanos (Kotliarenco, Muñoz, Gómez \& Aracena, 2009). Enfrentados a la oportunidad histórica que ofrece en Chile el sistema integral de protección a la infancia Chile Crece Contigo, es tiempo de generar conciencia respecto a la necesidad de garantizar recursos y conocimiento que permita dar cuerpo a la visión de una infancia segura y cuidada, bien tratada y adecuadamente estimulada (Consejo Infancia, 2006).

\section{Referencias}

Administration for Children and Families (2002). Making a difference in the lives of infants and toddlers and their families: The impacts of Early Head Start. Washington, DC: DHHS.

Appleyard, K., Egeland, B., van Dulmen, M. \& Sroufe, A. (2005). When more is not better: the role of cumulative risk in child behavior outcomes. Journal of Child Psychology and Psychiatry, 46, 3, p. 235-245.

Bedregal, P. (2006). Eficacia y efectividad en la atención de niños entre 0 y 4 años. Serie En Foco, $\mathrm{N}^{\circ}$ 79, Ed. Expansiva: Santiago de Chile.

Chazan_Cohen, R., Ayoub, C., Alexander Pan, B., Roggman, L., Raikes, H., McKelvey, L., Whiteside_Mansell, L. \& Hart, A. (2007). It Takes Time: Impacts of Early Head Start That Lead to Reductions in Maternal Depression Two Years Later. Infant Mental Health Journal, 28, 2, p. 151_170.

Cicchetti, D. \& Toth, S.L. (1995). A developmental psychopathology perspective on child abuse and neglect. Journal of American Academy of Child and Adolescent Psychiatry, 34, 5, p. 541-65.

Connell-Carrick, K. (2003). A Critical Review of the Empirical Literature: Identifying Correlates of Child Neglect. Child and Adolescent Social Work Journal, 20, 5, p. 389-425.

Consejo Infancia (2006). El Futuro de los Niños es Siempre Hoy: Propuestas del Consejo Asesor Presidencial para la Reforma de las Políticas de Infancia. Gobierno de Chile: Santiago de Chile. 
Cyrulnik, B. (2002). Los patitos feos. La resiliencia: una infancia infeliz no determina la vida. Barcelona: Gedisa.

Cyrulnik, B. (2003). El murmullo de los fantasmas. Volver a la vida después de un trauma. Barcelona: Gedisa.

Cyrulnik, B. (2007). De Cuerpo y Alma. Neuronas y afectos: la conquista del bienestar. Barcelona: Gedisa.

Cyrulnik, B. (2008). Autobiografía de un espantapájaros. Testimonios de resiliencia: el retorno a la vida. Barcelona: Gedisa.

Glaser, D. (2000). Child abuse and neglect and the brain—a review. Journal of Child Psychology and Psychiatry, 41, p. 97- 116.

Gray, R. \& McCormick, M.C. (2005). Early Childhood Intervention Programs in the US: Recent Advances and Future Recommendations. The Journal of Primary Prevention, 26, 3, p. 259_275.

Kalawski, J.P. \& Haz, A.M. (2003). Y ¿Dónde está la resiliencia? Una Reflexión Conceptual. Revista Interamericana de Psicología, 37, 2, p. 365-372.

Kotliarenco, M.A. \& Lecannelier, F. (2004). Resiliencia y coraje. El apego como mecanismo protector. A. Melillo, E. Suárez \& D. Rodríguez (comp.). Resiliencia y Subjetividad. Los ciclos de la vida. Buenos Aires: Paidós.

Kotliarenco, M.A. \& Pardo, M. (2003). Algunos Alcances sobre el sustento biológico de los comportamientos resilientes. Psykhe, 1, p. 119-124.

Kotliarenco, M.A., Cáceres, I. \& Fontecilla, M. (1997). Estado de Arte en Resiliencia. Organización Panamericana de la Salud.

Kotliarenco, M.A., Gómez, E. \& Muñoz, M. (2009). Centros de Desarrollo Infantil en Chile: Una Experiencia Piloto. Perspectivas - CEES UC, No 8.

Kotliarenco, M.A., Muñoz, M., Gómez, E. \& Aracena (2009). La Visita Domiciliaria en Programas de Intervención Temprana: una revisión de sus características, efectividad y desafíos. Manuscrito No Publicado.

Lecannelier, F. (2006). Apego e Intersubjetividad: Influencia de los Vínculos Tempranos en el Desarrollo Humano y la Salud Mental. LOM Ediciones: Santiago de Chile.

Lecannelier, F., Hoffman, M., Flores, F. \& Ascanio, L. (2008). Problemas, proyecciones y desafíos en la salud mental infantil: la necesidad de reformular el rol profesional. Horizonte de Enfermería, 19, 1, p. 1-9.

Leyton, E. \& Ferrer, S. (2007). Estudio Cualitativo de Evaluación. Proyecto Centros de Desarrollo Infantil Temprano. Informe de Resultados. Documento No Publicado. Fundación INTEGRA: Santiago de Chile.

Love, J., Banks, L., Raikes, H. \& Chazan Cohen, R. (2006). Head Start: What Do We Know About Its Effectiveness? What Do We Need to Know?, p. 550 575. K. McCartney \& D. Phillips (Eds). Blackwell Handbook of Early Childhood Development. Ed. Blackwell Publishing: Oxford, United Kingdom.

Luthar, S., Cicchetti, D. \& Becker, B. (2000). The Construct of Resilience: A Critical Evaluation and Guidelines for Future Work. Child Development, 71, 3, p. 543-562.

Massie, H. \& Campbell, B. (1983). The Massie-Campbell scale of mother-infant attachment indicators during stress (AIDS scale). Frontiers of Infant Psychiatry, J. Call, E. Galenson \& R. Tyson (comps). Nueva York: Basic Books, p. 294-412. 
National Evaluation of Sure Start Research Team (2008). National Evaluation Report: The Impact of Sure Start Local Programmes on Three Years Olds and Their Families. Institute for the Study of Children, Families and Social Issues, Birkbeck, University of London: London.

National Research Council (1993). Understanding Child Abuse and Neglect. National Academy Press: Washington, D.C.

Olds, D., Sadler, L. \& Kitzman, H. (2007). Programs for Parents of Infants and Toddlers: Recent Evidence from Randomized Trials. Journal of Child Psychology and Psychiatry, 48, p. 355_391.

Palmeros, M.J., Rojas, C. \& Traverso, R. (2008). Efectos del Programa Comunitario de Desarrollo Integral Temprano (DIT) en la depresión post natal de mujeres en trece comunas de la región metropolitana en las zonas nor poniente, centro y sur oriente en Santiago de Chile, 2006 - 2008. Seminario presentado a la Facultad de Psicología de la Universidad del Desarrollo para optar al título profesional de Psicólogo (a).

Rodríguez, S., Arancibia, V. \& Undurraga, C. (1976). Escala de Evaluación de Desarrollo Psicomotor para niños entre 0 y 2 años. Santiago: Editorial Galdoc.

Rutter, M. (1979). Protective factors in children's responses to stress and disadvantage. M.W. Kent \& J.E. Rolf (Eds.), Primary Prevention of Psychopathology, Vol.3: Social competence in children (pp. 49-74). Hanover, NH: University of New England Press.

Rutter, M. (1993). Resilience: Some conceptual considerations. Journal of Adolescent Health, 14, p. 626-631.

Rutter, M. (2006). Is Sure Start and Effective Preventive Intervention? Child and Adolescent Mental Health, 11, 3, p. 135_141.

Shonkoff, J. \& Phillips, D. (Eds) (2000). From Neurons to Neighborhoods: The Science of Early Childhood Development. Washington D.C.: National Academy Press.

Shore, A. (2001). The Effects of Early Relational Trauma on Right Brain Development, Affect Regulation, and Infant Mental Health. Infant Mental Health Journal, 22(1-2), p. 201-269.

Springer, K., Sheridan, J., Kuo, D. \& Carnes, M. (2007). Long-term physical and mental health consequences of childhood physical abuse: Results from a large population-based sample of men and women. Child Abuse \& Neglect, 31, p. 517-530.

Sroufe, L.A., Carlson, E.A., Levy, A.K., \& Egeland, B. (1999). Implications of attachment theory for developmental psychopathology. Development and Psychopathology, 11, p. 1-13.

Strasser, K. (2006). Evaluación de Programas de Intervención Temprana. Revista En Foco, Nro 78. Ed. Expansiva: Santiago de Chile.

Walsh, F. (2004). Resiliencia Familiar: estrategias para su fortalecimiento. Buenos Aires: Amorrortu. 